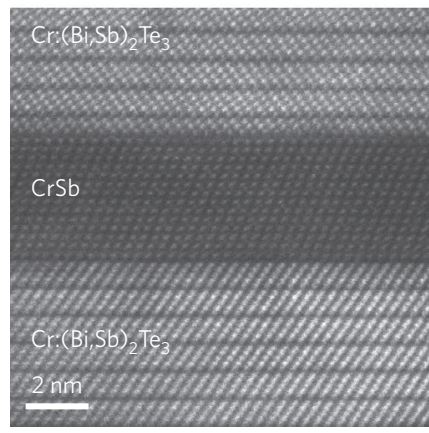

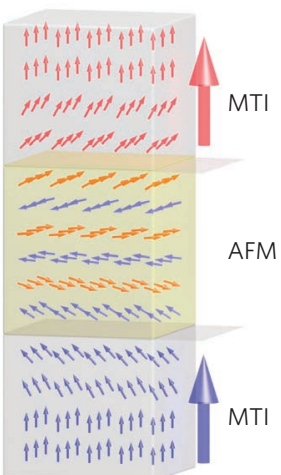

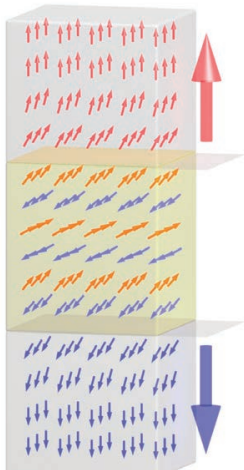

in-plane moments to pin the magnetic topological insulator at the interface, an overall parallel or antiparallel alignment of the (out-of-plane) moments of the ferromagnetic layers in a trilayer structure can be achieved, as illustrated in Fig. 2b. This is a nice example of a functional heterostructure that could be useful in engineering the quantum effects related to magnetic topological insulators.

There is no doubt that several materials challenges must be overcome before one will be able to observe the quantum anomalous Hall effect in these proximitycoupled systems at high temperatures; but the first steps have been taken, and there is more to come. In the near future, thorough magneto-transport studies, as well as angle-resolved photoemission spectroscopy, are needed to complete the experimental proof that the Dirac fermion becomes massive. As the topological surface state lives near the interface, we may see a similar surge of fascinating and surprising phenomena, as with complex oxide interfaces in the recent past $^{8}$. Emergent states are waiting to be discovered, and it is time for the topological insulators to stack up.

materials are well lattice-matched, leading to abrupt interfaces as shown in Fig. 2a, and this makes $\mathrm{CrSb}$ (whose Néel temperature is about $700 \mathrm{~K}$ ) one of the few antiferromagnets suited to incorporation into functional layer structures. In this system, $\mathrm{He}$ and colleagues demonstrate that the magnetic topological insulator can be exchangecoupled to the antiferromagnet, leading to an enhancement of the exchange field up to $\sim 50 \mathrm{mT}$, an increase in the Curie temperature from $\sim 38 \mathrm{~K}$ to $\sim 90 \mathrm{~K}$, and an enhancement of the coercive field to $67 \mathrm{mT}$ (for a bilayer sample, as compared with $47 \mathrm{mT}$ for a single layer). Interestingly, by using the antiferromagnet with its

Thorsten Hesjedal and Yulin Chen are at the Clarendon Laboratory, Department of Physics, University of Oxford, Parks Road, Oxford OX1 3PU, UK.

e-mail:Thorsten.Hesjedal@physics.ox.ac.uk; Yulin.Chen@physics.ox.ac.uk

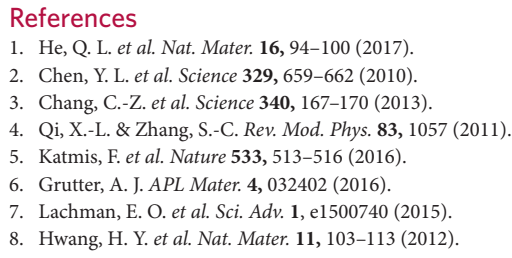

\title{
PEROVSKITE PHOTOVOLTAICS
}

\section{Slow recombination unveiled}

One of the most salient features of hybrid lead halide perovskites is the extended lifetime of their photogenerated charge carriers. This property has now been shown experimentally to originate from a slow, thermally activated recombination process.

\section{Jacques-E. Moser}

n just four years, hybrid organicinorganic lead halide perovskites have transitioned from a scientific curiosity known only by a handful of specialists into the most desirable and most studied semiconductor materials in the field of photovoltaics ${ }^{1,2}$. The reason for such interest is two-fold: this type of material is quite cheap and simple to produce; and the power conversion efficiency of simple, solution-processed solar cells based on perovskite layers has increased rapidly from an initial promising value of $9 \%^{1}$ to now more than $22 \%^{3}$. In addition, the open-circuit voltage for perovskite cells 
commonly approaches the thermodynamic limit by exceeding $1.2 \mathrm{~V}$. Several chemical and physical investigations, as well as theoretical calculations, have been applied to the prototype perovskite compound methylammonium lead triiodide $\left(\mathrm{MAPbI}_{3}\right)$, thereby highlighting the key properties of the material. Among these are long charge carrier lifetime and diffusion length, which have been intriguing and somewhat unexpected. In fact, $\mathrm{MAPbI}_{3}$ is characterized by a strong absorption constant and a high photoluminescence quantum yield, which indicates a directbandgap transition. However, the lifetime of photogenerated carriers exceeds the Langevin limit for direct recombination by several orders of magnitude 4 . Various possible rationales for this apparent contradiction have been proposed on the basis of experimental data and theoretical predictions. Recently, calculations of the band structure that take into account spin-orbit coupling or the positional disorder of organic cations in the perovskite lattice have suggested the presence of a slightly indirect bandgap ${ }^{5-8}$. Yet no direct experimental evidence has been reported that could support the idea of a phonon-assisted recombination process for photocarriers.

Now, writing in this issue of Nature Materials, Eline Hutter and colleagues ${ }^{9}$ report time-resolved microwave conductance and photoluminescence experiments carried out at various temperatures. Their observations show that the non-geminate electron-hole recombination following photoexcitation of $\mathrm{MAPbI}_{3}$ perovskite is a thermally activated process, which is consistent with a slow phonon-mediated pathway. This behaviour is found to be general for solution-processed perovskite films in the tetragonal phase, and is compatible with theoretical predictions for the mixed direct-indirect bandgap character of this semiconductor material ${ }^{5}$.

The direct or indirect character of the bandgap transition in a semiconductor is commonly derived experimentally by analysing the absorption spectrum of the solid. A direct (vertical) intrinsic transition involves the excitation of an electron from the material's fully occupied valence band to the unoccupied conduction band by an incident photon (Fig. 1a). In an indirect (non-vertical) transition, the photoexcitation of valence-band electrons is only possible through the involvement of lattice phonons, whose energy and momentum are either borrowed from or released to the solid (Fig. 1b). Since the need for a phonon contribution makes

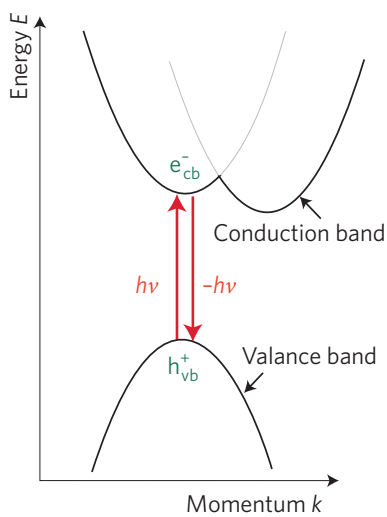

b

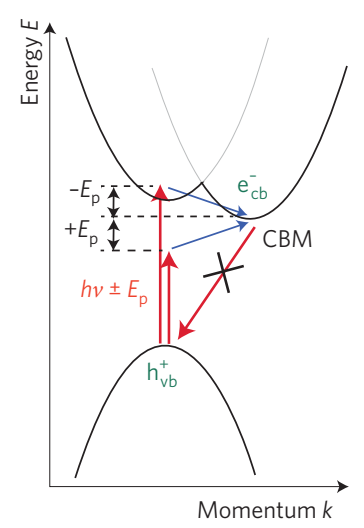

c

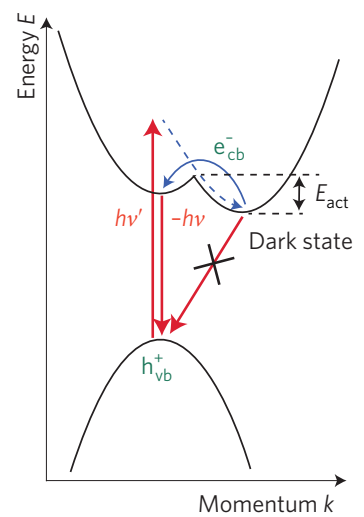

Figure 1 | Schematic band diagram of a semiconductor. In this simplified representation, the valence and conduction bands appear as parabolas, with the electron energy $E$ being a quadratic function of its momentum $k$. a, Since photons of energy $h v$ (where $h$ is Planck's constant and $v$ is the photon frequency) carry only a very weak momentum, $k$ should remain unchanged during an electronic transition. A vertical transition following the Franck-Condon principle $(\Delta k=0)$ is called a direct transition. A transition in the opposite direction is equally allowed, leading generally to a fast radiative recombination $(-h v)$ of photogenerated electrons $\left(\mathrm{e}_{\mathrm{cb}}^{-}\right)$and holes $\left(\mathrm{h}_{\mathrm{vb}}^{+}\right) . \mathbf{b}$, An indirect transition to a minimum of the conduction band (CBM) that is shifted in the $k$ dimension implies that a vibration of the solid lattice (phonon) is coupled to the electronic movement. The change in momentum $(\Delta k>0)$ is brought by the absorption or release of a phonon of energy $E_{\mathrm{p}}$ in the solid. A non-vertical transition from the CBM back to the valence band maximum is forbidden, and no radiative recombination of photogenerated carriers is generally observed in this case. c, Scheme of the recombination pathway proposed by Hutter and colleagues 9 . Following light absorption with $h v^{\prime} \geq 1.7 \mathrm{eV}$, photogenerated hot electrons relax down to a dark state (dashed curved arrow). Radiative electron-hole recombination $(-h v)$ implies that an activation barrier of energy $E_{\text {act }}$ must be initially overcome and some momentum exchanged during a phonon-mediated process (solid curved arrow).

indirect transitions less probable, the absorption constant is usually $2-3$ orders of magnitude less than that of a directbandgap material. In $\mathrm{MAPbI}_{3}$ perovskite, both direct and indirect transitions can apparently take place simultaneously. In addition, an excitonic band appears as a shoulder on the red side of the bandgap absorption threshold, and artefacts due to reflectance have been frequently observed. As a result, interpreting the intrinsic absorption spectrum of this material is quite difficult.

In their analysis, Hutter and colleagues recognized that light absorption leading to the generation of charge carriers in $\mathrm{MAPbI}_{3}$ might take place mainly through a direct transition, with the radiative recombination of the charges still following an indirect mechanism. Concentrating on the latter process, they monitored its kinetics using a combination of timeresolved microwave conductance and photoluminescence measurements. Data produced by both techniques concurred to show that the lifetime of free mobile charge carriers is extended by lowering the temperature, which is in contrast with the behaviour observed in other directbandgap semiconductors such as GaAs.
The temperature dependence followed an Arrhenius-type relationship, from which an activation energy of $E_{\text {act }}=47 \mathrm{meV}$ was extracted.

This effect could be attributed to the fast localization of electrons in shallow traps, whose energetic levels would lie below the conduction band edge. However, this is ruled out by microwave conductance spectra, which show that the mobility of carriers is a maximum when the material is irradiated just above the direct bandgap. The general picture proposed by Hutter and colleagues to explain these results is shown in Fig. 1c: electrons photoexcited in the conduction band thermally relax into dark states in band-like structures, yielding highly mobile carriers. Fast radiative recombination of these electrons with valence-band holes is forbidden, thus resulting in extended charge carrier lifetime. This situation is analogous to the case of an indirect-bandgap semiconductor, for which radiative electron-hole recombination is momentum-forbidden (Fig. 1b).

These results offer a rationale for the extended carrier lifetime in organo-lead halide perovskites in the tetragonal form, and are suggestive of an indirect bandgap in this material. However, more detailed 
information on the proposed mechanism will be required before it can be exploited in the design of new high-performance materials. In particular it is unclear whether the observed dark states are indeed populated 'directly' through an indirect transition. The nature of the phonons involved, the possible role of polarons, and the potential for multiple conduction and valence bands still need to be scrutinized. Besides, it is yet to be explained why fast non-radiative recombination does not seem to occur from the dark states, in contrast with the case of the orthorhombic, low-temperature phase of $\mathrm{MAPbI}_{3}$. As shown by Hutter and colleagues, direct access to the mobility of carriers is essential for understanding the details of their dynamics, which, along with a better timeresolution, might be offered by broadband time-resolved terahertz spectroscopy. Further studies using this technique could allow us to unravel the fast processes that occur during the thermalization of photocarriers. They could also provide a handle for identifying the origin of lowfrequency vibrations that are seemingly at the heart of the unique properties of lead halide perovskites.

Jacques-E. Moser is at the Institute of Chemical Sciences \& Engineering of the École Polytechnique
Fédérale de Lausanne, CH-1015 Lausanne, Switzerland.

e-mail:je.moser@epfl.ch

\section{References}

1. Lee, M. M., Teuscher, J., Miyasaka, T., Murakami, T. N. \& Snaith, H. J. Science 338, 643-647 (2012).

2. Kim, H.-S. et al. Sci. Rep. 2, 591 (2012).

3. Saliba, M. et al. Energ Environ. Sci 9, 1989-1997 (2016).

4. Johnston, M. B. \& Herz, L. M. Acc. Chem. Res. 49, 146-154 (2016).

5. Brivio, F., Butler, K. T., Walsh, A. \& van Schilfgaarde, M. Phys. Rev. B 89, 155204 (2014).

6. Motta, C. et al. Nat. Commun. 6, 7026 (2015).

7. Zheng, F., Tan, L. Z., Liu, S. \& Rappe, A. M. Nano Lett. 15, 7794-7800 (2015).

8. Etienne, T., Mosconi, E. \& De Angelis, F. J. Phys. Chem. Lett. 7, 1638-1645 (2016)

9. Hutter, E. M. et al. Nat. Mater. 16, 115-120 (2017).

\section{A RECIPE FOR GROWTH}

"Everything is the way it is because it got that way." If D'Arcy Thompson voiced this aphorism, commonly attributed to him, he was probably thinking of how the form of objects can be understood from the growth law that produced them: the idea expounded in his seminal book On Growth and Form, which has its centenary this year. But it is also a statement of historical contingency and path dependence: to understand the way things are, you need to consider the detailed dynamics of formation.

Equilibrium thermodynamics challenges that, asserting instead that the form of a structure corresponds to that of lowest free energy in a pre-existing and predictable energy landscape; dynamics is just the journey to a predetermined destination. But in the real world, complex systems with heterogeneous components often fail to reach that platonic ideal even when they grow under close-to-equilibrium conditions ${ }^{1}$. That might seem to condemn us, in such cases, to making structures riddled with uncontrolled disorder and defects. But might it in fact be possible to control and direct non-equilibrium growth dynamics so as to access a greater diversity of structures than equilibrium offers?

Whitelam et al. have argued that this should be feasible in both one-dimensional polymerizationtype processes ${ }^{2}$, and two- and threedimensional self-assembly ${ }^{3}$. They considered two kinds of building blocks self-assembling stochastically via interactions weak enough to permit disassembly too, under conditions driving the system out of equilibrium. This could apply, for example, to structures self-assembled by colloidal forces, hydrogen-bonding, DNA hybridization, or coordination chemistry in metal-organic frameworks. The out-of-equilibrium driving force determining the growth rate can be tuned to switch between extreme segregation and mixing of the building blocks (Whitelam et al. considered binary mixtures), with some control over the domain size.

Nguyen and Vaikuntanathan have now generalized this approach to propose some design principles for controlled non-equilibrium self-assembly ${ }^{4}$. Their approach is to specify a Hamiltonian for their target structure that represents the equilibrium state of that structure for a set of monomer units with interaction strengths (more precisely, lattice-model coupling constants) rescaled from those that exist in the actual system being studied. The question is then how to tweak the driving forces for self-assembly - in this case, the chemical potentials of the various components, which are proportional to concentration, although other dials should be both necessary and possible - so as to realize this effective Hamiltonian.

Nguyen and Vaikuntanathan show that such trajectories can be calculated in principle for the $1 \mathrm{D}$ and $2 \mathrm{D}$ systems considered by Whitelam et al.,

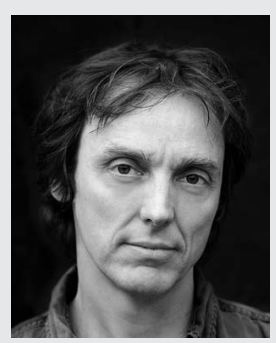

PHILIP BALL

supplying a chemical-potential protocol for achieving a given target structure. They think it should be possible, for example, to switch crystal growth from face-centred cubic to body-centred cubic, or perhaps even to quasicrystalline. The proof will, as ever, be in the experimental pudding.

If such a strategy for controlling non-equilibrium growth via concentration exists, we might wonder whether nature exploits it already. Specific polymer structures there are generally achieved not by stochastic growth but by templating. But might this be the key to, say, nature's apparent ability to direct the polymorphism of calcium carbonate biominerals ${ }^{5}$ ?

References

1. Kim, A. J., Scarlett, R., Biancaniello, P. L., Sinno, T. \& Crocker, J. C. Nat. Mater. 8, 52-55 (2009).

2. Whitelam, S., Schulman, R. \& Hedges, L. Phys. Rev. Lett. 109, 265506 (2012).

3. Whitelam, S., Hedges, L. O. \& Schmidt, J. D. Phys. Rev. Lett. 112, 155504 (2014).

4. Nguyen, M. \& Vaikuntanathan, S. Proc. Natl Acad. Sci. USA http://doi.org/bt6d (2016).

5. Cartwright, J. H., Checa, A. G., Gale, J. D., Gebauer, D. \& Sainz-Díaz, C. I. Angew. Chem. Int. Ed. 51, 11960-11970 (2012). 